\title{
A successful case of multiple stereotactic radiosurgeries for ipsilateral recurrent trigeminal neuralgia
}

\author{
Emily Daugherty, MD, ${ }^{1}$ Shripal Bhavsar, MD, MBA, ${ }^{2}$ Seung Shin Hahn, MD, ${ }^{1}$ Daniel Bassano, PhD, ${ }^{1}$ \\ and Walter Hall, MD, MBA ${ }^{3}$
}

\begin{abstract}
Departments of ${ }^{1}$ Radiation Oncology and ${ }^{3}$ Neurosurgery, SUNY Upstate Medical University, Syracuse, New York; and ${ }^{2}$ Department of Radiation Oncology, Integris Cancer Institute, Oklahoma City, Oklahoma
\end{abstract}

\begin{abstract}
Trigeminal neuralgia is a common pain syndrome primarily managed medically, although many patients require surgical or radiotherapeutic intervention. Stereotactic radiosurgery has become a preferred method of treatment given its high efficacy rates and relatively favorable toxicity profile. However, many patients have refractory pain even after repeat courses of stereotactic radiosurgery. Historically, 2 courses have been the limit in such patients. The authors present a case of multiply recurrent trigeminal neuralgia treated with a third course of radiosurgery in which the patient had successful pain control and no additional toxicity. Meticulous attention to the therapeutic technique allows the continued application of stereotactic radiosurgery in patients.
\end{abstract}

http://thejns.org/doi/abs/10.3171/2014.9.JNS13959

KEY WORDS trigeminal neuralgia; repeat stereotactic radiosurgery; radiosurgery; Gamma Knife; repeat Gamma Knife; functional neurosurgery; pain

$\mathrm{T}$ RIGEMINAL neuralgia (TN) is a peripheral neuropathy characterized by intermittent episodes of severe facial pain originating in the sensory nucleus of cranial nerve $\mathrm{V}$, the trigeminal nerve. According to the International Headache Society, the condition is defined by the following: paroxysmal attacks lasting from $1 \mathrm{sec}-$ ond to 2 minutes and affecting 1 or more divisions of the trigeminal nerve (V1-V3); intense, sharp, superficial, or stabbing pain precipitated by specific triggers or actions; attacks characteristic for the individual patient; no clinically evident neurological deficit; and pain not attributed to another disorder. ${ }^{12}$ The etiology of TN is not completely understood; however, it has been suggested that enlargement of the superior cerebellar artery branches may act to compress the trigeminal nerve as it exits the pons, disrupting the integrity of the myelin sheath and transmitting sensory input with very slight stimulation. This hyperactivity to minor sensation is perceived as intense pain. Clinically, patients, who usually present in the 6th decade, will most often complain of unilateral, shooting facial pain that is intense and episodic. Trigeminal neuralgia affects women more often than men. ${ }^{5}$ Patients may have spontaneous pain or certain triggers for pain, including chewing, talking, teeth brushing, shaving, face touching, or cold stimuli. Patients will often demonstrate a pattern of avoidance behaviors, and in severe instances, the disorder can lead to dramatic weight loss. Subsequent workup yields normal neurological examination findings, and when performed, MRI is negative for structural compression via bone or tumors in classic TN. The role of imaging in patients with TN is somewhat controversial, as large evidence-based studies have provided inconsistent results and varying degrees of sensitivity and specificity. Thus, the American Academy of Neurology and the European Federation of Neurological Societies (AAN/EFNS) has concluded that there is insufficient evidence to support or refute the utility of MRI to identify neurovascular compression in classic TN. ${ }^{11}$ If routine imaging studies are obtained, underlying structural etiologies for the pain will appear in about $15 \%$ of patients with TN. In certain demyelinating disorders such as multiple sclerosis, demyelination of the trigeminal nerve can be seen, although some instances reveal vascular compression as well..$^{20}$

Current available treatment options include medical management, surgical intervention, or stereotactic radiosurgery. Pain control is initiated using medication, most often an anticonvulsant. Medical treatment options commonly include carbamazepine as the initial drug of choice, but other anticonvulsants, such as gabapentin, phenytoin, oxcarbazepine, lamotrigine, and topiramate, may be used as well. ${ }^{5}$ Often patients will poorly tolerate the medication or have pain refractory to medical management. In

ABBREVIATIONS BNI = Barrow Neurological Institute; GKRS = Gamma Knife radiosurgery; $\mathrm{MVD}=$ microvascular decompression; $\mathrm{TN}=$ trigeminal neuralgia . SUBMITTED March 26, 2014. ACCEPTED September 30, 2014.

INCLUDE WHEN CITING Published online March 27, 2015; DOI: 10.3171/2014.9.JNS13959.

DISCLOSURE The authors report no conflict of interest concerning the materials or methods used in this study or the findings specified in this paper. 
this setting, the patient can have more invasive treatment such as surgical intervention, which is classified as either destructive to the trigeminal nerve or nondestructive. Destructive procedures include rhizotomy, balloon compression, and radiofrequency thermocoagulation. ${ }^{22}$ Rhizotomy involves injecting an agent directly into the affected nerve branch. In balloon compression a small balloon can be inserted into the nerve branches to compress the nerve within a bony foramen. Common side effects of the aforementioned surgical options include sensory loss (50\%), dysesthesias (6\%), anesthesia dolorosa (4\%), and corneal numbness (4\%). ${ }^{11,22,30}$ Another, often more favorable, nondestructive surgical option is microvascular decompression (MVD). This procedure often leads to very good, sustained pain relief and is highly recommended for younger patients or those with few to no comorbidities. While the risk of morbidity is known to be low, MVD remains a major neurosurgical procedure that involves craniotomy and can require days of inpatient recovery postprocedure. Side effects include CSF leakage, hearing loss, hemorrhage, numbness, and facial weakness. Complications most often include aseptic meningitis (11\%) and hearing loss (10\%), as well as sensory loss (7\%). ${ }^{11}$ High-dose stereotactic radiosurgery has been used since the 1970s and is very effective for the treatment of TN. In patients with recurrent or refractory pain, repeat radiosurgery has also been found to be effective. Two courses are often the limit as there have been concerns about tolerance of the brainstem, as well as the nerve itself, to further radiotherapy. Radiation-induced trigeminal nerve or pontine necrosis and anesthesia dolorosa are both feared complications among radiosurgery practitioners.

In this case report we describe the interesting course of a young patient who underwent 3 separate Gamma Knife radiosurgery (GKRS) courses to the same nerve for the successful treatment of refractory TN. We also discuss the literature, including a similar report, and highlight favorable characteristics and situations in which multiple GKRSs can be performed safely and with positive outcomes.

\section{Case Report}

History and Examination

A 38-year-old man initially presented to his primary care physician 14 years earlier, reporting a 3-year history of right jaw and tongue pain. He described his pain as "a nail being stuck into my tongue and jaw." The pain lasted anywhere from a few seconds up to half a minute maximum. The frequency of his pain was irregular, occurring as much as several times in a day to as infrequently as 2-3 times a week. Shaving, eating, or talking usually elicited pain. The patient was healthy without any neurological or other medical conditions. At the time, MRI was performed, which incidentally revealed a left clival mass, which was biopsied 2 months later and was diagnosed as Paget's disease. He remained asymptomatic of the Paget's disease and was placed on $200 \mathrm{mg}$ of hydroxyzine 3 times a day. He did not achieve any relief, however, and was placed on $200 \mathrm{mg}$ of carbamazepine 3 times a day for a year after presenting to his primary physician. He contin- ued to require increasing doses of the carbamazepine and ultimately underwent balloon compression 16 months after presentation to his primary physician. He reported that his initial neurosurgeon, who worked at an outside facility, deferred MVD as the first-line treatment both because of its invasive nature and because of neurosurgeon preference. The balloon compression relieved his pain, and he required no medication until, unfortunately, the pain returned about 6 months later. He was again placed on carbamazepine at the prior dosage, although it was eventually raised to $300 \mathrm{mg} 3$ times a day with very minimal relief of his symptoms. His neurosurgeon then referred him to radiation oncology. At the time of initial presentation to our department, 15 months had passed since his surgery, 10 of which he was symptomatic. After a detailed discussion, he elected to undergo GKRS.

\section{First GKRS and Postoperative Course}

He received $85 \mathrm{~Gy}$ to the $100 \%$ isodose line to the proximal trigeminal nerve in two 4-mm shots, the first of which was immediately adjacent to the lateral pons. Following GKRS, the patient had significant pain relief, and at the 1-month follow-up, he was slowly decreasing his pain medications (200 mg 2 times a day). Available physical examination follow-up reports noted "very mild diminished sensation along the body of the mandible."

Unfortunately, 5 months after GKRS, his pain recurred and, in fact, was more severe. His carbamazepine dose was raised to $800 \mathrm{mg}$ daily without any abatement. The pain, which was rated $1-2 / 10$ with episodes up to $10 / 10$, was unresponsive to any medications or intervention. Since he had sustained relief after the initial GKRS with minimal response to medical management, the decision was made to proceed with another GKRS.

\section{Second GKRS and Postoperative Course}

At 6 months after the first GKRS, a total of 70 Gy was administered to the $100 \%$ isodose line in two 4-mm shots just distal to the site of emergence of the right trigeminal nerve. Following the second GKRS, the patient did very well with only occasional twinges of pain, which, according to the patient, never returned to pre-procedure levels. The diminished sensation remained stable without further neurological symptoms. Neurological examination was essentially unremarkable, except for an area of perceived numbness from the lateral canthus to the lateral zygomatic arch in the V2 distribution, with no other sensory, motor, or cerebellar deficits. Corneal reflex testing was intact bilaterally. There was no symptom or sign of facial weakness, decrease in hearing, or ocular dryness. There were no signs of deafferentation pain syndrome. He was seen annually for 7 years without any change or increase in symptoms. Ultimately, 9 years after the second GKRS, the patient had 10/10, severe lancinating pain that recurred in the right-sided roof of the mouth, radiating through to the right mandible. He reported right facial numbness, which at the time was severe and located over the right zygomatic arch, along with paresthesia-like sensations around the right eye. There was neither facial asymmetry nor deficit in the facial muscles; corneal reflex testing remained intact. At the time, the patient was not agreeable 
to restarting medications given their side effects. Since he had long-term pain relief after the second GKRS, relative intolerance to medications, and the possibility of treating the nerve more distally, he underwent a third course of GKRS.

\section{Third GKRS and Postoperative Course}

The right trigeminal nerve was treated with a single 4-mm shot delivering a dose of 70 Gy to the $100 \%$ isodose line. To avoid overlap with prior treatments, the target was made to the anterior portion of the right trigeminal nerve root. The composite dose delivered is pictured in Fig. 1. Upon follow-up 1 year later, the patient was without pain and medication free. The minimal numbness in the right side of his face was still present along the V2 sensory distributions and had not increased in severity. There were no signs of deafferentation, anesthesia dolorosa, corneal reflex impairment, or facial muscle paralysis. According to the patient, numbness to light touch in the above distribution was not bothersome or progressing. At the 3-year follow-up after the third GKRS, the patient was pain free and without medications; however, he now noticed the minor decreased sensation to light touch along the V1 distribution as well, just posterior to the right lateral side of the hairline. During the patient's 5-, 113-, and 36-month pain-free durations after each GKRS, his Barrow Neurological Institute (BNI) Facial Hypesthesia Scale score was never above II, as he had mild facial numbness that was not bothersome..$^{33}$ A table describing both the latency until pain relief after each surgical and GKRS procedure, as well as the duration of pain relief achieved for each of these procedures, can be seen in Table 1 .

This patient underwent 3 GKRS courses to the same nerve with continued duration of pain response and minimal side effects.
TABLE 1. Summary of procedures performed in a patient with recurrent TN

\begin{tabular}{lllc}
\hline \multicolumn{1}{c}{ Treatment } & Date & $\begin{array}{c}\text { Latency Until } \\
\text { Pain Relief }\end{array}$ & $\begin{array}{c}\text { Duration of Effect } \\
\text { (mos) }\end{array}$ \\
\hline Balloon compression & 2000 & NA $^{*}$ & 6 \\
\hline GKRS of $85 \mathrm{~Gy}$ & 2001 & Immediate & 5 \\
\hline GKRS of $70 \mathrm{~Gy}$ & 2002 & $1-2$ wks & 113 \\
\hline GKRS of $70 \mathrm{~Gy}$ & 2011 & $1 \mathrm{mo}$ & $36 \dagger$ \\
\hline
\end{tabular}

NA $=$ not available.

* Not available because procedure was performed at an outside institution.

† Until most recent follow-up in August 2014.

\section{Discussion}

The pathophysiology of TN has been presumed attributable to vascular compression in the root entry zone of the trigeminal nerve in the midpons, leading to subsequent demyelination. This theory has led to the development of MVD surgery, in which a retromastoid craniectomy is performed to relieve pressure on the nerve..$^{18}$ Multiple surgical interventions are now possible, including glycerol rhizotomy, radiofrequency ablation, and percutaneous balloon compression; however, surgical intervention is commonly considered secondary to medical interventions because of surgical morbidities. ${ }^{34}$ While MVD is the most invasive treatment option, it remains the least likely to damage the nerve. As with surgery, medical management can have significant side effects, including bone marrow suppression and hepatic dysfunction in addition to cognitive impairment.

Stereotactic radiosurgery has emerged as an effective therapeutic alternative with comparatively decreased side effects. Lars Leksell originally described GKRS as a suc-

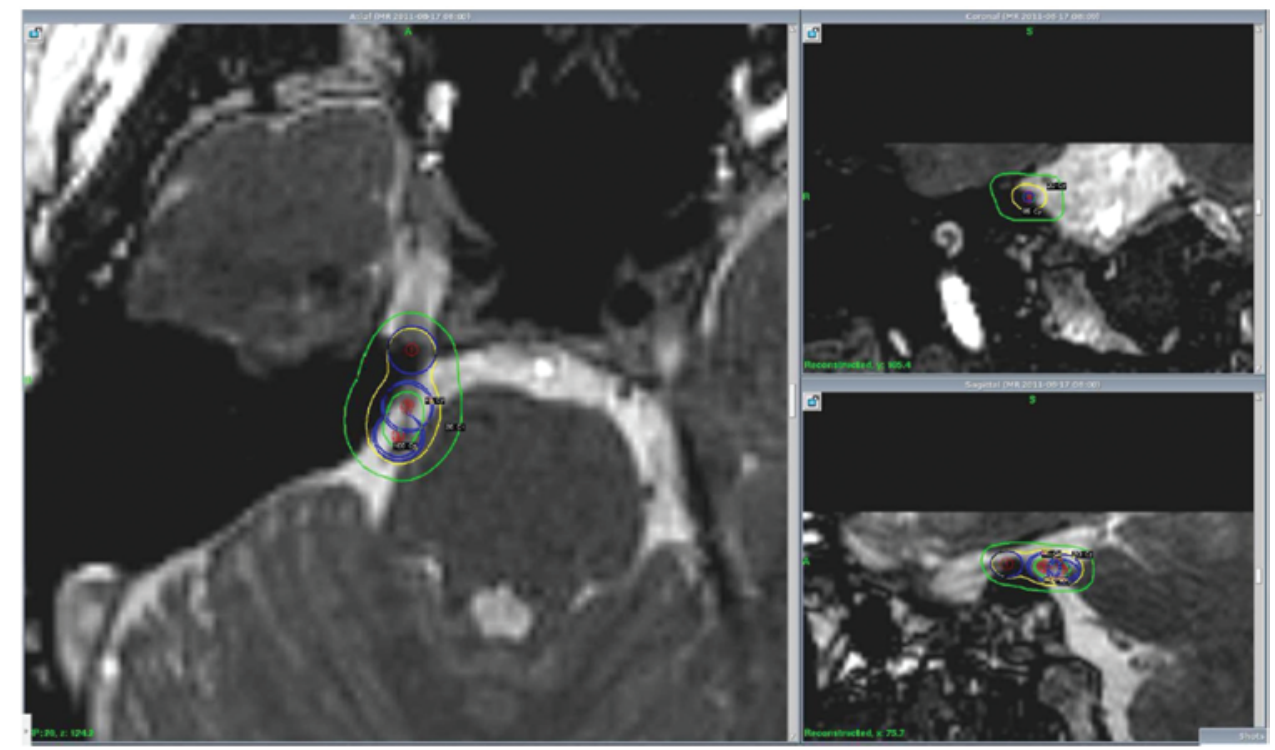

FIG. 1. The composite dose delivered in 3 treatments via GKRS is depicted. The shot distribution along the path of the trigeminal nerve root is also shown in multiple planes. The initial 2 treatments delivered 2 shots to the nerve root, both proximal to the brainstem. The third treatment was a single shot to the region distal to the prior treatments. Blue, yellow, and green lines represent isodose lines. Figure is available in color online only. 
cessful treatment for patients with TN in $1971 . .^{5}$ The efficacy of GKRS for TN pain is believed to be attributable to the induction of axonal degeneration. Doses upwards of 80-100 Gy can produce visible axonal degeneration and edema of the trigeminal nerve. Currently, GKRS plans commonly use targets involving the nerve entry zone rather than the gasserian ganglia, the trigeminal nerve cell bodies. Previously, studies utilizing targeting of the trigeminal ganglion had very poor rates of pain relief and mild success rates. $5,16,28$ The belief behind targeting the root entry zone is that this area involves a transition zone in the axons from peripheral to central myelin, which in theory is believed to be more susceptible to radiation. ${ }^{33}$ Several articles have documented histopathological evidence of axonal degeneration and fibrosis after GKRS. ${ }^{1,15}$

Trigeminal neuralgia treatment responses are evaluated on the standardized BNI pain intensity scale, in which scores of I-III are considered good control. ${ }^{5}$ Data arranged in Chin et al. revealed that after GKRS up to 93\% of patients experience durable pain relief ranging from no trigeminal pain to durable pain control on medication. In a comparison of 8 trials evaluating TN outcomes for initial GKRS, the percentage of patients achieving good control ranged from $69 \%$ to $94 \%$, with up to $72 \%$ of patients requiring no medications.

Gamma Knife radiosurgery continues to be a safe, efficacious treatment for both medically and surgically refractory TN. ${ }^{9,17}$ Refractory TN presents a difficult clinical situation, as it is well established that a patient's initial surgical intervention remains the most successful, with subsequent surgical interventions offering diminishing returns. Overall, the success of repeat GKRS with regard to pain relief is estimated to be around $60 \%-70 \%$ in numerous studies. ${ }^{10,25}$ However, patients undergoing GKRS have better response rates if they have not undergone a prior surgical intervention. ${ }^{23}$ Studies on the GKRS doses used for TN have reported that doses $\geq 70$ Gy provide better pain control than doses $<70$ Gy. Doses over 100 Gy are likely to result in trigeminal nerve necrosis..$^{15}$ Some institutions will use 2 isocenters to treat a larger target volume of the trigeminal nerve. In a 2005 study from our institution, we found that 2-isocenter dose plans were associated with significantly greater improvements in the BNI score than 1-isocenter dose plans, with no increase in neurological morbidities. ${ }^{2}$ Updated published results revealed an increased response rate with dose escalation and 2 isocenters. ${ }^{21}$ While these results differ from those in a prospective randomized controlled trial by Flickinger et al., the difference is likely attributable to a difference in isocenter placement. ${ }^{8}$ Morbidini-Gaffney et al. reported greater overlap of isocenters than the 3- to 5-mm separation described by Flickinger et al., which allowed a shorter nerve length and/or volume to be treated to a higher dose. ${ }^{21}$ Again, low rates of tolerable numbness and paresthesias continued to occur in the Morbidini-Gaffney et al. study compared with the rates seen by Pollock et al. (11\% vs $20 \%$, respectively). ${ }^{27}$

Few studies have long-term data on patient outcomes and quality of life following GKRS for TN. An article from the University of Maryland with a 5-year follow-up has reiterated the above-described findings, reporting a larger number of patients achieving durable pain control if they had not undergone prior surgical procedures. Response duration was significantly better for those who had no prior invasive treatment than for those in whom a previous surgical intervention had failed (32 vs 21 months, $p$ $<0.02) .{ }^{6}$ The duration of an initial response after GKRS is a significant factor predicting the level of response to subsequent GKRS. 13,26,32

There is very limited experience in the literature regarding patients receiving a third course of GKRS for refractory TN. To our knowledge, only 2 other papers have described patients with a third course of GKRS for TN..$^{10,14}$ Gellner et al. reports on 2 patients who had each undergone a third and fourth GKRS treatment with single isocenters; however, very little information is reported about these patients. ${ }^{10}$ One patient underwent a third GKRS 2 years after a second radiosurgical procedure and a fourth GKRS 12 months later. The other patient also underwent a third GKRS 2 years after a second and later underwent thermal coagulation and a subsequent fourth GKRS in a 5 -year period. There are no other available data regarding these patients' clinical outcomes.

A paper by Jones et al. details the case of a 72-yearold man who underwent a third GKRS, a case that has some similarities with our patient. ${ }^{14}$ The authors describe a patient who failed initial medical management as well as radiofrequency gangliotomy between his first and second GKRS. Initially, he was treated with 42 Gy to the trigeminal root entry zone to the $50 \%$ isodose line (max point dose $84 \mathrm{~Gy}$ ). Failed pain control was documented 2 months after the initial GKRS. A second radiosurgical procedure was performed 11 months after the initial GKRS. Treatment was again directed to the proximal nerve root entry zone, but a dose of 27 Gy to the $50 \%$ isodose line was delivered (max point dose $54 \mathrm{~Gy}$; a 36\% dose reduction). The patient had 6.5 months of pain relief on no medications and underwent a third GKRS 17 months after his initial GKRS. The third treatment involved dose delivery to a target "millimeters distal to the prior target" and prescribed 20 Gy to the $50 \%$ isodose line (max point dose of $40 \mathrm{~Gy}$ ). As in our case, a more distal target was chosen to decrease neural toxicity and the risk of necrosis. However, after 3 symptom-free months, pain again recurred and was treated surgically with MVD. The authors reported on a 6-month follow-up from the last GKRS in their conclusion, with a total of 26 months since the patient's initial GKRS treatment.

The present case report and the one by Jones et al. are the only 2 articles to date that provide detailed information on the treatments and outcomes in patients who have undergone a third GKRS treatment. However, there are significant differences between these 2 cases. In our patient, there was only 1 surgical treatment prior to the first GKRS. Jones et al. describes the need for 2 surgical interventions, each due to GKRS failure-one between the first and second GKRS and one after the third GKRS. As noted previously, GKRS has improved effectiveness with a lower number of prior surgical interventions. ${ }^{4}$ Although the initial surgical treatment failed in our patient, he continued to have longer periods of pain control between each GKRS and did not require any interventions or medica- 
tions after the third GKRS. Importantly, a longer followup after the final treatment was furnished in our case report, which impacts the reporting of late toxicities.

Considering the technical aspects, our treatment plans differed from those described by Jones et al. in that we continued to use high doses in each subsequent radiosurgical procedure, with 2 isocenters continued in the second GKRS and moving to 1 isocenter with an anterior shift of the target in the third GKRS to avoid necrosis. The distance between shots was $3 \mathrm{~mm}$ for the initial 2 treatments. The third course involved delivery of a single shot $5 \mathrm{~mm}$ anterior to prior treatments at the entrance to Meckel's cave. The maximum point dose to the trigeminal nerve was located midway between the brainstem and the gasserian ganglion, and it was estimated at $150 \mathrm{~Gy}$, with the patient receiving most of this dose from the first 2 treatments. The accumulated dose to the gasserian ganglion was estimated at 50-70 Gy, with most of the dose having been contributed by the third GKRS. Despite repeated high doses to the nerve, there was no increase in bothersome or significant facial numbness or paresthesias. Importantly, our patient had no side effects or necrosis in the brain. However, concern about pontine necrosis motivated us to place the third course shot quite anterior to the prior shots. In full, the composite maximum point dose to the brainstem was 108.7 Gy. According to Chin and Regine, patients who undergo repeat GKRS have higher incidences of facial numbness, but they report no increase in dysesthesias or radiation necrosis, which was demonstrated in our case. ${ }^{5}$

A significant concern in treating our patient was deafferentation pain or anesthesia dolorosa. According to an article by Pollock, radiosurgery should be classified as a destructive procedure, and the success of GKRS is directly related to the degree of trigeminal nerve injury. ${ }^{24}$ These assertions have been reiterated in the literature. ${ }^{27,29,31}$ Therefore, sufficient damage is required to achieve lasting pain relief, but this increased injury must be balanced with the greater risk of deafferentation syndromes. Anesthesia dolorosa is experienced as intractable severe pain in an area of numbness. This chronic pain syndrome is very difficult to control and is believed to be attributable to deafferentation-releasing second-order neurons, which generate spontaneous pain signals without stimulus..$^{30}$ Albeit rare, anesthesia dolorosa can result from both surgical and radiosurgical therapies for $\mathrm{TN}$ with different incidences. For patients who are status post-trigeminal rhizotomy, incidence is estimated at $2.4 \% .{ }^{30} \mathrm{In}$ our review, extrapolating from articles in which patients with TN were treated with GKRS and there were documented cases of anesthesia dolorosa, we estimate the risk of anesthesia dolorosa to be between $0.005 \%$ and $0.03 \% .^{3,19}$ The patient in our case report did not develop anesthesia dolorosa and has had continued pain relief following 3 GKRS procedures, with an estimated total dose of 150 Gy to the trigeminal nerve. A 2004 abstract from ASTRO (American Society for Radiation Oncology) reported that repeat GKRS with median cumulative doses upwards of 180 Gy produced successful results with a higher propensity to be off medications than at lower doses with no increase in deafferentation pain incidence. ${ }^{7}$ Nevertheless, it remains that the data on anes- thesia dolorosa are scarce in the literature, which limits an accurate estimation of risk.

\section{Conclusions}

In summary, we present a case in which the trigeminal nerve was treated using multiple courses of GKRS with minimal toxicity and continuing effectiveness. Important considerations in offering further GKRS treatment to patients with $\mathrm{TN}$ refractory to previous radiosurgical procedures include response to medical management, number of prior surgical interventions, and duration of response to prior GKRS. Technical aspects of treatment delivery should be meticulously defined and may need adjustment from the standard protocol. A shot can be placed anteriorly to reduce the risk of trigeminal or brainstem necrosis without needing to reduce dose and compromise treatment effectiveness. A well-selected patient can be safely treated with multiple courses of GKRS without decreasing subsequent target doses.

\section{References}

1. Al-Otaibi F, Alhindi H, Alhebshi A, Albloushi M, Baeesa S, Hodaie M: Histopathological effects of radiosurgery on a human trigeminal nerve. Surg Neurol Int 4 (Suppl 6):S462S467, 2013

2. Alpert TE, Chung CT, Mitchell LT, Hodge CJ, Montgomery CT, Bogart JA, et al: Gamma knife surgery for trigeminal neuralgia: improved initial response with two isocenters and increasing dose. J Neurosurg 102 Suppl:185-188, 2005

3. Aubuchon AC, Chan MD, Lovato JF, Balamucki CJ, Ellis TL, Tatter SB, et al: Repeat gamma knife radiosurgery for trigeminal neuralgia. Int J Radiat Oncol Biol Phys 81:1059-1065, 2011

4. Brisman R: Repeat gamma knife radiosurgery for trigeminal neuralgia. Stereotact Funct Neurosurg 81:43-49, 2003

5. Chin LS, Patel S, Mattingly T, Kwok Y: Trigeminal neuralgia, in Chin LS, Regine WF (eds): Principles and Practice of Stereotactic Radiosurgery. New York: Springer, 2008, pp 519-526

6. Dhople AA, Adams JR, Maggio WW, Naqvi SA, Regine WF, Kwok Y: Long-term outcomes of Gamma Knife radiosurgery for classic trigeminal neuralgia: implications of treatment and critical review of the literature. Clinical article. J Neurosurg 111:351-358, 2009

7. Dutta PR, Kwok Y, Herman JM, Chin LS, Colliver C, St Clair WH, et al: Comparison of repeat GK-SRS for refractory or recurrent trigeminal neuralgia: does dose matter. Int J Radiat Oncol Biol Phys 60 (Suppl):S405, 2004

8. Flickinger JC, Pollock BE, Kondziolka D, Phuong LK, Foote RL, Stafford SL, et al: Does increased nerve length within the treatment volume improve trigeminal neuralgia radiosurgery? A prospective double-blind, randomized study. Int J Radiat Oncol Biol Phys 51:449-454, 2001

9. Gardner WJ: Trigeminal neuralgia. Clin Neurosurg 15:156,1968

10. Gellner V, Kurschel S, Kreil W, Holl EM, Ofner-Kopeinig P, Unger F: Recurrent trigeminal neuralgia: long term outcome of repeat gamma knife radiosurgery. J Neurol Neurosurg Psychiatry 79:1405-1407, 2008

11. Gronseth G, Cruccu G, Alksne J, Argoff C, Brainin M, Burchiel K, et al: Practice parameter: the diagnostic evaluation and treatment of trigeminal neuralgia (an evidence-based review): report of the Quality Standards Subcommittee of the American Academy of Neurology and the European Federation of Neurological Societies. Neurology 71:1183-1190, 2008 
12. Headache Classification Subcommittee of the International Headache Society: The International Classification of Headache Disorders: 2nd edition. Cephalalgia 24 Suppl 1:9-160, 2004

13. Herman JM, Petit JH, Amin P, Kwok Y, Dutta PR, Chin LS: Repeat gamma knife radiosurgery for refractory or recurrent trigeminal neuralgia: treatment outcomes and quality-of-life assessment. Int J Radiat Oncol Biol Phys 59:112-116, 2004

14. Jones GC, Elaimy AL, Demakas JJ, Jiang H, Lamoreaux WT, Fairbanks RK, et al: Feasibility of multiple repeat gamma knife radiosurgeries for trigeminal neuralgia: a case report and review of the literature. Case Rep Med 2011:258910, 2011

15. Kondziolka D, Lacomis D, Niranjan A, Mori Y, Maesawa S, Fellows W, et al: Histological effects of trigeminal nerve radiosurgery in a primate model: implications for trigeminal neuralgia radiosurgery. Neurosurgery 46:971-977, 2000

16. Lindquist C, Kihlström L, Hellstrand E: Functional neurosurgery - a future for the gamma knife? Stereotact Funct Neurosurg 57:72-81, 1991

17. Loeser JD, Calvin WH, Howe JF: Pathophysiology of trigeminal neuralgia. Clin Neurosurg 24:527-537, 1977

18. Lunsford LD, Apfelbaum RI: Choice of surgical therapeutic modalities for treatment of trigeminal neuralgia: microvascular decompression, percutaneous retrogasserian thermal, or glycerol rhizotomy. Clin Neurosurg 32:319-333, 1985

19. Maesawa S, Salame C, Flickinger JC, Pirris S, Kondziolka D, Lunsford LD: Clinical outcomes after stereotactic radiosurgery for idiopathic trigeminal neuralgia. J Neurosurg 94:14-20, 2001

20. Meaney JF, Watt JW, Eldridge PR, Whitehouse GH, Wells JC, Miles JB: Association between trigeminal neuralgia and multiple sclerosis: role of magnetic resonance imaging. $\mathbf{J}$ Neurol Neurosurg Psychiatry 59:253-259, 1995

21. Morbidini-Gaffney S, Chung CT, Alpert TE, Newman N, Hahn SS, Shah H, et al: Doses greater than 85 Gy and two isocenters in Gamma Knife surgery for trigeminal neuralgia: updated results. J Neurosurg 105 Suppl:107-111, 2006

22. Obermann M: Treatment options in trigeminal neuralgia. Ther Adv Neurol Disord 3:107-115, 2010

23. Petit JH, Herman JM, Nagda S, DiBiase SJ, Chin LS: Radiosurgical treatment of trigeminal neuralgia: evaluating quality of life and treatment outcomes. Int J Radiat Oncol Biol Phys 56:1147-1153, 2003

24. Pollock BE: Radiosurgery for trigeminal neuralgia: is sensory disturbance required for pain relief? J Neurosurg 105 Suppl:103-106, 2006

25. Pollock BE, Foote RL, Link MJ, Stafford SL, Brown PD, Schomberg PJ: Repeat radiosurgery for idiopathic trigeminal neuralgia. Int J Radiat Oncol Biol Phys 61:192-195, 2005
26. Pollock BE, Foote RL, Stafford SL, Link MJ, Gorman DA, Schomberg PJ: Results of repeated gamma knife radiosurgery for medically unresponsive trigeminal neuralgia. J Neurosurg 93 Suppl 3:162-164, 2000

27. Pollock BE, Phuong LK, Gorman DA, Foote RL, Stafford SL: Stereotactic radiosurgery for idiopathic trigeminal neuralgia. J Neurosurg 97:347-353, 2002

28. Rand RW, Jacques DB, Melbye RW, Copcutt BG, Levenick MN, Fisher MR: Leksell Gamma Knife treatment of tic douloureux. Stereotact Funct Neurosurg 61 (Suppl 1):93-102, 1993

29. Rogers CL, Shetter AG, Fiedler JA, Smith KA, Han PP, Speiser BL: Gamma knife radiosurgery for trigeminal neuralgia: the initial experience of The Barrow Neurological Institute. Int J Radiat Oncol Biol Phys 47:1013-1019, 2000

30. Sandwell SE, El-Naggar AO: Nucleus caudalis dorsal root entry zone lesioning for the treatment of anesthesia dolorosa. J Neurosurg 118:534-538, 2013

31. Shetter AG, Rogers CL, Ponce F, Fiedler JA, Smith K, Speiser BL: Gamma knife radiosurgery for recurrent trigeminal neuralgia. J Neurosurg 97 (5 Suppl):536-538, 2002

32. Wang L, Zhao ZW, Qin HZ, Li WT, Zhang H, Zong JH, et al: Repeat gamma knife radiosurgery for recurrent or refractory trigeminal neuralgia. Neurol India 56:36-41, 2008

33. Xu Z, Schlesinger D, Moldovan K, Przybylowski C, Sun X, Lee CC, et al: Impact of target location on the response of trigeminal neuralgia to stereotactic radiosurgery. J Neurosurg 120:716-724, 2014

34. Zakrzewska JM, Akram H: Neurosurgical interventions for the treatment of classical trigeminal neuralgia. Cochrane Database Syst Rev (9):CD007312, 2011

\section{Author Contributions}

Conception and design: Daugherty, Bhavsar, Hahn. Acquisition of data: Daugherty, Bhavsar, Bassano. Analysis and interpretation of data: Daugherty, Bhavsar, Bassano. Drafting the article: Daugherty, Bhavsar. Critically revising the article: Daugherty, Bhavsar, Hahn, Hall. Reviewed submitted version of manuscript: all authors. Administrative/technical/material support: Daugherty, Bhavsar, Bassano. Study supervision: Hahn, Hall.

\section{Correspondence}

Emily Daugherty, Department of Radiation Oncology, SUNY Upstate Medical University, 750 E. Adams St., Syracuse, NY 13210. email: emily.cupelo@gmail.com. 